\title{
The Boundary Element Analysis of Wave Force acting on Multiple Cylinders
}

\author{
+ Namhyeong Kim, Tan Ngooc Than Cao* and Soonbo Yang** \\ †, *, ** Department of Civil Engineering/Marine \& Environmental Research Institute, \\ Jeju National University, Jeju 690-756, Korea
}

\begin{abstract}
In this paper, the boundary element method is applied to solve the diffraction of waves by multiple vertical cylinders under the assumption of linear wave theory. A numerical analysis by boundary element method is based on Green's theorem and introduced to an integral equation for the fluid velocity potential around the cylinders. The numerical results obtained in this study are compared with the experimental data and the results of the theory using multiple scattering techniques. The comparisons show strong agreement. This numerical analysis method developed by using boundary element method could be used broadly for the design of various offshore structures to be constructed in coastal zones in the future.
\end{abstract}

Key words : diffraction of waves, boundary element method, multiple vertical cylinders, green's theorem, wave forces

\section{Introduction}

The interaction between waves and multiple vertical cylinders phenomenon can be reviewed briefly as follows. As the incident waves impinge on each cylinder, the reflected waves move outward. On the sheltered side of the cylinder there will be a "shadow" zone where the wave fronts are bent around the cylinder, the $\mathrm{so}^{-}$called diffracted wave. The reflected waves and diffracted waves, combined, are usually called the scattered waves. The scattered waves of each cylinder can affect other cylinders. This process is generally termed diffraction. By this process, the pressure around the cylinders will change and therefore the forces on the cylinders will be influenced.

There have been several studies dealing with the interaction of linear water waves with multiple cylinders. Twersky(1950) constructed a solution using an iterative procedure in which successive scatters by each of the cylinders were introduced at each order. This method was extended to the water wave case by Ohkusu(1974). The main drawback of the iterative procedure is that it rapidly becomes unmanageable as the number of cylinders increases. Spring and Monkmeyer(1974) proposed a solution for the interaction of water waves with the cylinders using eigenfuntion expansion approach. They formulated the problem in terms of a matrix equation and the solution is obtained by the inversion of the matrix. Chakrabarti(1978) extended the work of Spring and Monkmeyer(1974), and obtained the solution for the diffracted wave of multiple cylinders by carrying out the analysis in a complex domain. Subsequently, Linton and Evans(1990) made a major simplification to the theory proposed by Spring and Monkmeyer(1974). In addition, Sobierajski(1970) presented experiment data for the interaction of waves with an infinite row of equally spaced cylinders at different spacing.

Notably, based on the boundary element method, wave force, run-up and free-surface elevation around a single circular vertical cylinder and various arrays of two to three of vertical circular cylinders were analyzed numerically by authors(Kim et al., 2007; Kim and Cao, 2008a; 2008b).

In this paper, a numerical analysis method by boundary element method is presented to calculate wave forces acting on each of multiple vertical cylinders namely, four vertical circular cylinders in square and lozenge array. The numerical analysis method is developed by using Green's function in direct boundary element method and introduced to an integral equation for the fluid velocity potential around the multiful cylinders. To verify, the results obtained from this study were compared with the experiment data obtained by Sobierajski(1970), the results of Chakrabarti(1978), and the results of Linton and Evans(1990). The comparisons are in good agreement.

\section{Basic equations}

In this study, the interaction of waves with four vertical

\footnotetext{
† Corresponding author, nhkim@jejunu.ac.kr 064)754-3452

* ctnthan@jejunu.ac.kr 064)754-3453

** beeo17@hanmail.net 064)754-3453
} 
circular cylinders in square and lozenge array is considered under the assumption of linear potential flow. The cylinders, having radius $a$, are arranged in the water of uniform depth $h$. The global Cartesian coordinate system $(x, y, z)$ is defined with the origin located on the still-water level, the $z$ axis directed vertically upwards, $x$ and $y$ axis directed horizontally as shown in Fig. 1.

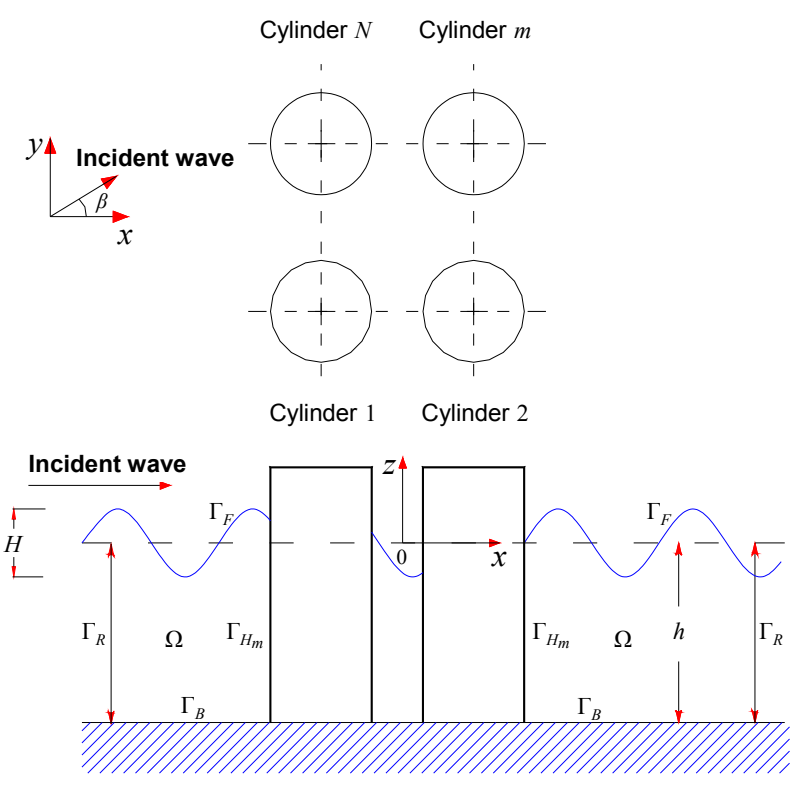

Fig. 1 Definition of multiple vertical cylinders

It is assumed that the fluid is inviscid, incompressible, its motion is irrotational, and fluid motion is small. The cylinders subjected to a train of regular waves of height $H$ and angular frequency $\sigma$ propagating at an angle $\beta$ to the positive $x$ axis. The velocity potential $\Phi(x, y, z ; t)$ can be defined by:

$$
\Phi(x, y, z ; t)=R_{e}\left[\phi(x, y, z) e^{-i \sigma t]}\right.
$$

where $R_{e}$ [ ] denotes the real part of a complex expression.

In equation (1), the total velocity potential is defined as follows:

$$
\begin{aligned}
& \phi=\phi_{i}+\phi_{s} \\
& \phi_{i}=-i \frac{g}{\sigma} \frac{H}{2} \varphi_{i}, \phi_{s}=-i \frac{g}{\sigma} \frac{H}{2} \varphi_{s} \\
& \varphi_{i}=\frac{\cosh k(h+z)}{\cosh k h} e^{i k(x \cos \beta+y \sin \beta)}
\end{aligned}
$$

where $\phi_{i}$ and $\phi_{s}$ are incident wave velocity potential and scattered velocity potential, respectively. $H / 2$ is wave amplitude, $g$ is the acceleration due to gravity, and the wave number $k$ is the positive real root of the dispersion relation:

$$
\sigma^{2}=g k \tanh k h
$$

Boundary value problems by the formulation of scattered wave velocity potential $\varphi_{s}$ are given as follows:

- Laplace equation:

$$
\nabla^{2} \varphi_{s}=0 \quad \text { in } \Omega
$$

- Free surface boundary condition:

$$
\frac{\partial \varphi_{s}}{\partial z}-\frac{\sigma^{2}}{g} \varphi_{s}=0 \quad \text { on } \Gamma_{F}
$$

- Cylinder surface boundary condition:

$$
\frac{\partial \varphi_{s}}{\partial n}=-\frac{\partial \varphi_{i}}{\partial n} \quad \text { on } \Gamma_{H_{m}}
$$

- Sea bed boundary condition:

$$
\frac{\partial \varphi_{s}}{\partial z}=0 \quad \text { on } \Gamma_{B}
$$

- Radiation boundary condition:

$$
\lim _{R \rightarrow \infty} \sqrt{R}\left\{\frac{\partial \varphi_{s}}{\partial R}-i k \varphi_{s}\right\}=0 \quad \text { on } \Gamma_{R}
$$

where $\Omega$ is fluid region, $\Gamma_{F}$ is free surface, $\Gamma_{H_{m}}$, $m=1,2, \ldots, N$ is the body surfaces of the cylinders, $\Gamma_{B}$ is the sea bed, $\Gamma_{R}$ is the vertical boundary at infinity, $n$ is the normal drawn outwardly on the boundary, $i$ is the imaginary unit $i=\sqrt{-1}$, and $R=\sqrt{x^{2}+y^{2}}$.

The incident wave velocity potential $\varphi_{i}$ and scattered wave velocity potential $\varphi_{s}$ are defined as follows:

$$
\begin{aligned}
& \varphi_{i}=\frac{\cosh k(h+z)}{\cosh k h} \Psi_{i}, \Psi_{i}=e^{i k(x \cos \beta+y \sin \beta)} \\
& \varphi_{s}=\frac{\cosh k(h+z)}{\cosh k h} \Psi_{s}(x, y)
\end{aligned}
$$

If the definitions of equations (7) and (8) are substituted into equations $(6 . \mathrm{a}) \sim(6 . e)$, the boundary values with $\Psi_{s}$ are obtained as follows:

$\nabla^{2} \Psi_{s}+k^{2} \Psi_{s}=0 \quad$ in $\Omega$

$$
\frac{\partial \Psi_{s}}{\partial n}=-\frac{\partial \Psi_{i}}{\partial n} \quad \text { on } S_{H_{m}}
$$




$$
\lim _{R \rightarrow \infty} \sqrt{R}\left\{\frac{\partial \Psi_{s}}{\partial R}-i k \Psi_{s}\right\}=0 \quad \text { on } S_{\infty}
$$

In equations (9.a) (9.c) boundary value problems are two-dimension problem of $x-y$ plane as shown in Fig. 2. Finally, because of analyzing boundary value problems by $\Psi_{s}$, the scattered wave velocity potential is calculated. Thus, the total velocity potential, wave pressure, and wave force can be calculated by using it.

The formulation and discretization by boundary element method were mentioned in detail(Kim and Cao, 2008a; 2008b).

$$
* j
$$

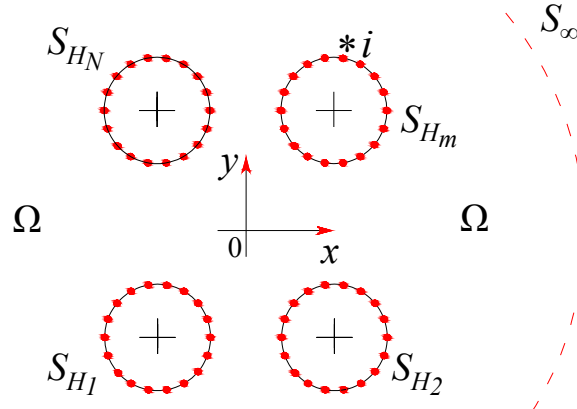

Fig. 2 Configuration of numerical model

\section{Numerical analysis and remarks}

To confirm the validity of this numerical analysis method and to investigate the effect of the neighboring cylinders on the wave forces acting on each cylinder, three different arrays of multiple vertical circular cylinders namely, seven cylinders in one line normal to the incident waves and four cylinders in square and lozenge array as shown in Fig. 3, are considered respectively.

To verify the numerical results of this study is demonstrated through the comparison with the experimental data obtained by Sobierajski(1970) and the results obtained from the theory proposed by Chakrabarti(1978). In the author's knowledge, Sobierajski(1970) tested interaction of regular waves (for $k a=0.1$ ) with a large single cylinder in a finite tank width to represent an infinite row of equally spaced cylinders at different spacing. These experimental (a)

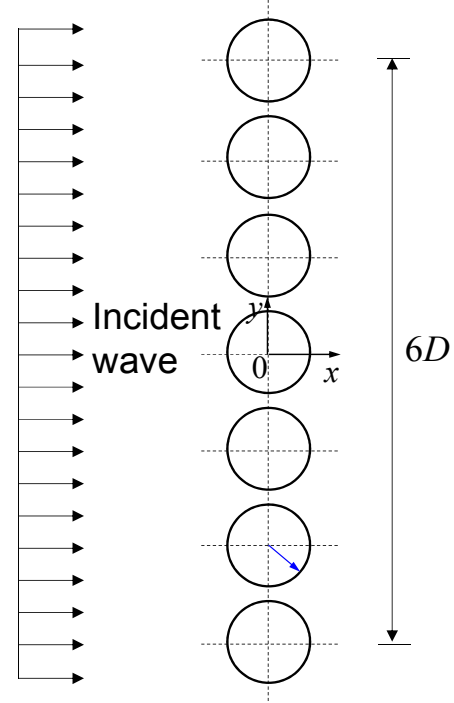

(b)

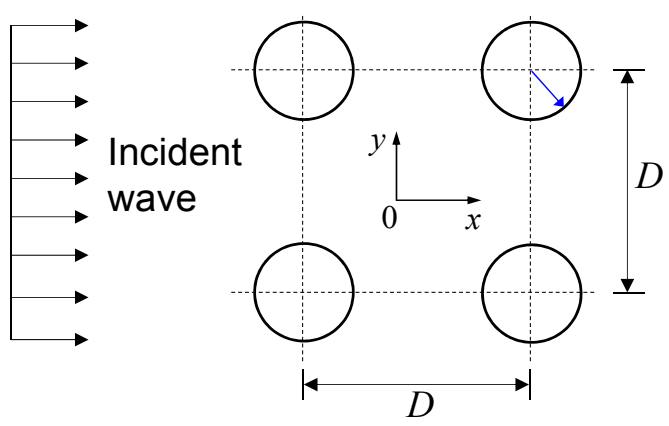

(c)

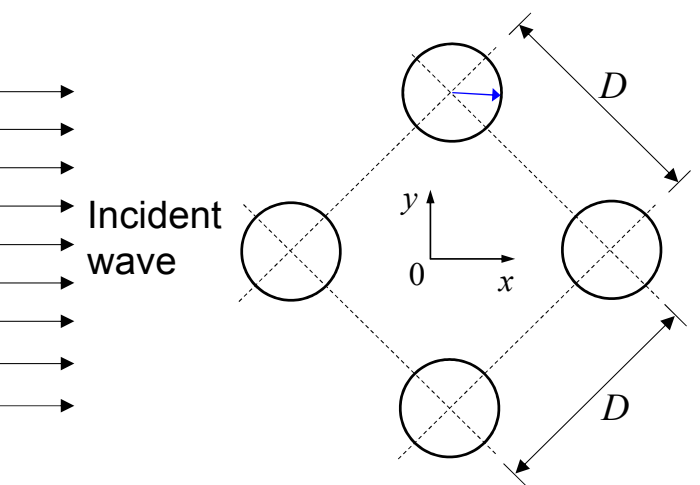

Fig. 3 Geometries for: (a) Seven cylinders in tandem normal to the incident wave; (b) Four cylinders in square array; (c) Four cylinders in lozenge array

data are compared with the results obtained from this study as shown in Fig. 4. The results of this method are obtained for seven equally spaced cylinders $(N=7)$ in a line normal to the incident waves (see Fig. 3a). Fig. 4 presents a non-dimensional form of the force ratio $f_{F}$ versus the distance ratio $\gamma=2 a / D$. In which, $f_{F}$ is the ratio of the maximum wave force on a cylinder in the row seven cylinders to the corresponding maximum wave force on the isolate cylinder. $D$ is the distance between the centers of 
the cylinders. $\gamma=1$ represents that the cylinders are touching each other, whereas $\gamma=0$ means that the distance among the cylinders $D \rightarrow \infty$. Also in Fig. 4, the results obtained by Chakrabarti(1978) are plotted for comparison. The comparisons show the strong agreement.

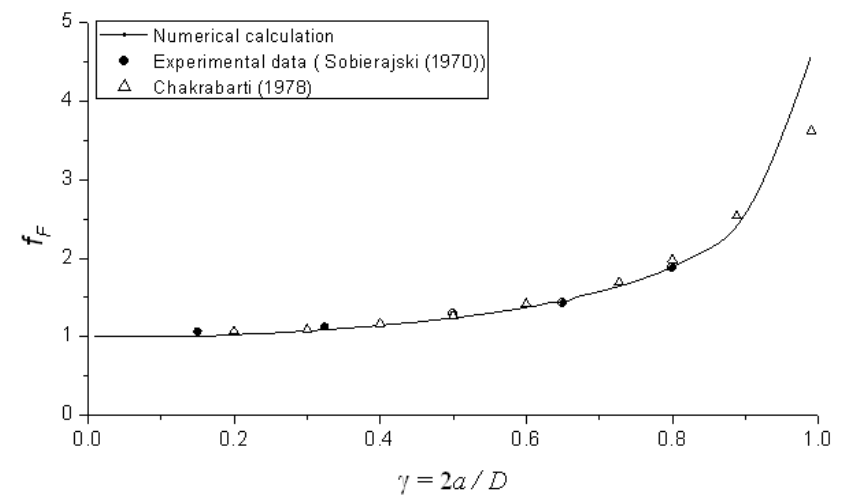

Fig. 4 Force ratio $f_{F}$ versus cylinder spacing ratio $\gamma$ for seven cylinders in tandem normal to incident wave for $k a=0.1$

(a)

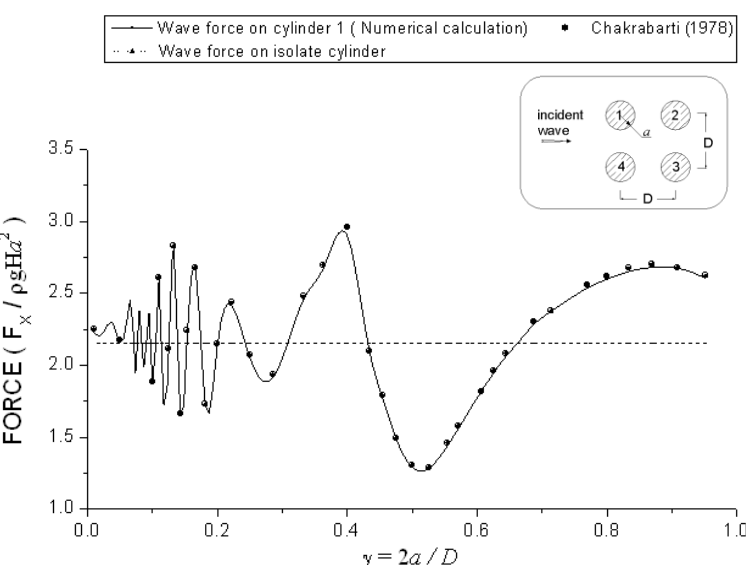

(b)

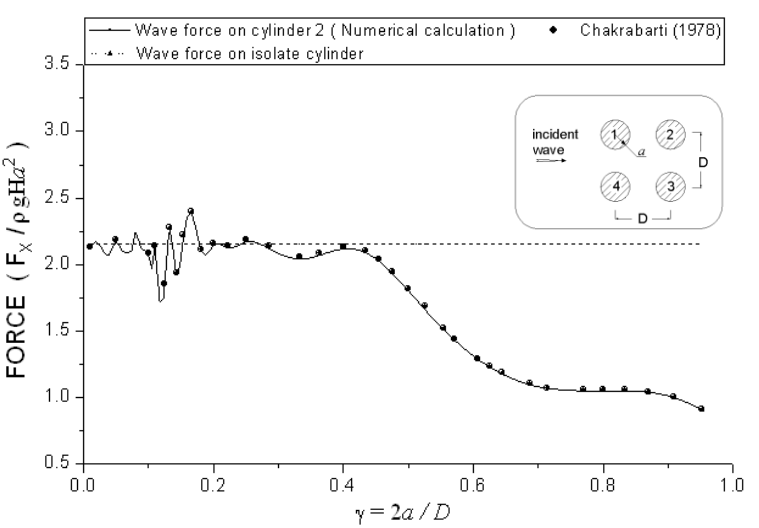

Fig. 5 Wave forces in $x$-direction on each cylinder in square array for $k a=0.1$ : (a) Wave force on cylinder 1; (b) Wave force on cylinder 2 (a)

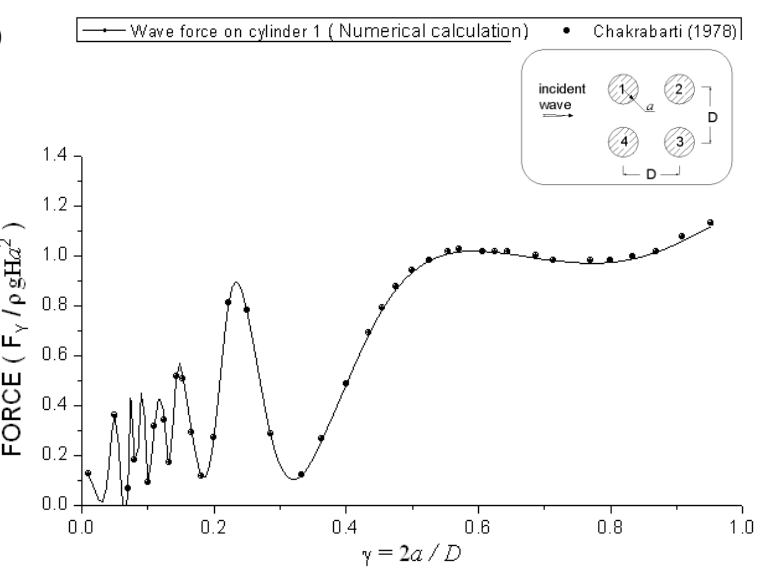

(b)

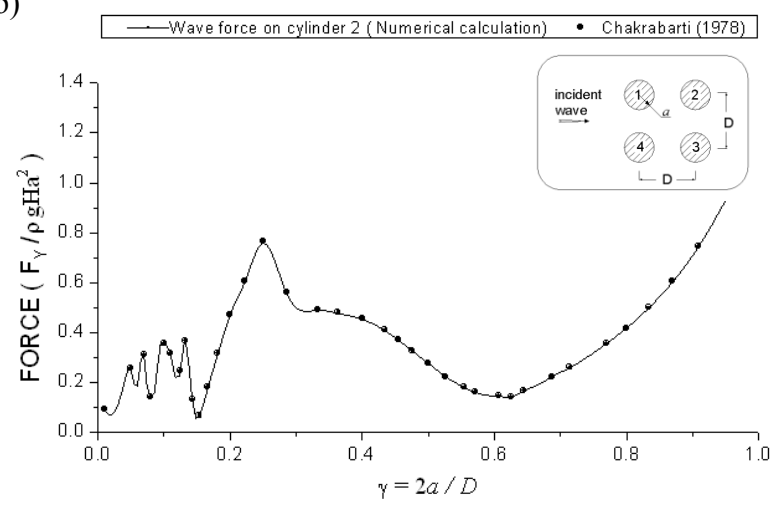

Fig. 6 Wave forces in $y$-direction on each cylinder in square array for $k a=0.1$ : (a) Wave force on cylinder 1; (b) Wave force on cylinder 2

In addition, to investigate the effect of the neighboring cylinders on the wave forces acting on each of cylinders, two different geometries are considered in this study namely, four cylinders in square and lozenge array (see Fig. $3 \mathrm{~b}$ and 3c). In all figures, the wave forces are nondimensionalized by $\rho g \mathrm{Ha}^{2}$ and the magnitude of run-up are nondimensionalized by $H$.

From Fig. 5 to Fig. 8, wave forces in $x$ - and $y$-direction acting on each of four cylinders in square and lozenge array versus ratio $\gamma=2 a / D$ are presented. In order to compare with the results of Chakrabarti(1978), the wave forces are presented for $h / a=10, k a=1.0$. Due to the symmetry of the geometry, the wave forces on the cylinder 1 and 2 in square array are presented. In lozenge array, the wave forces in $x$ - and $y$-direction on the cylinder 2 and 4 are the same. The wave forces in $y$-direction on the cylinder 1 and 3 are zero. The wave force on the isolate cylinder is also plotted for the purpose of comparison. The computed results show that as the distance between the cylinders increases, the wave force on each cylinder of four-cylinder group oscillates around the wave force on an 
Namhyeong Kim, Tan Ngooc Than Cao and Soonbo Yang

(a)

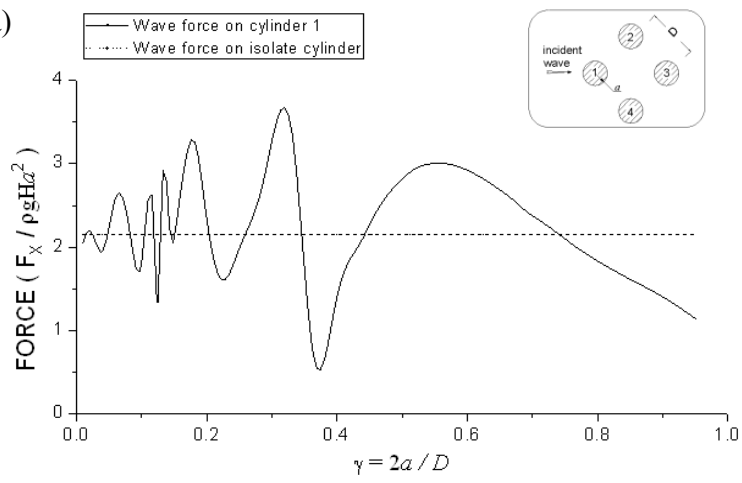

(b)

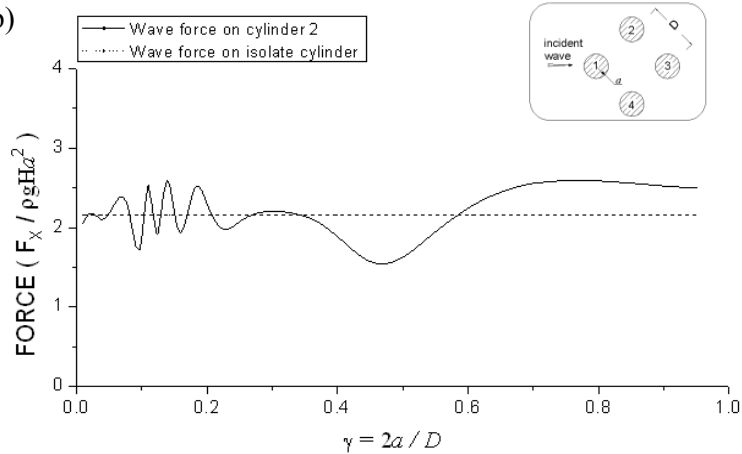

(c)

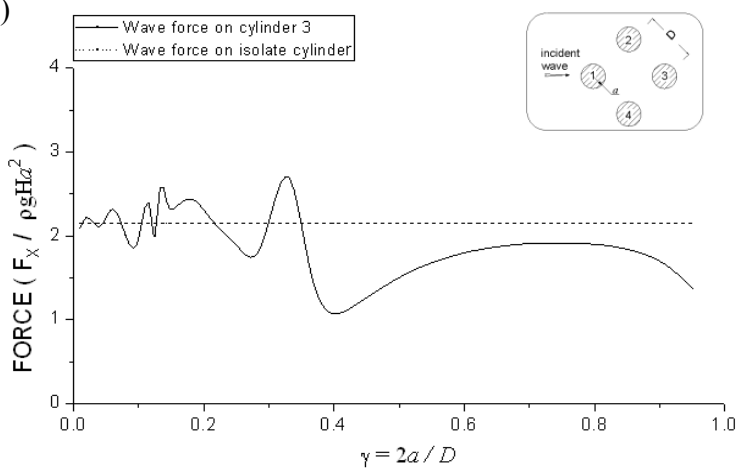

Fig. 7 Wave forces in $x$-direction on each cylinder in lozenge array for $k a=1.0$ : (a) Wave force on cylinder 1; (b) Wave force on cylinder 2; (c) Wave force on cylinder 3

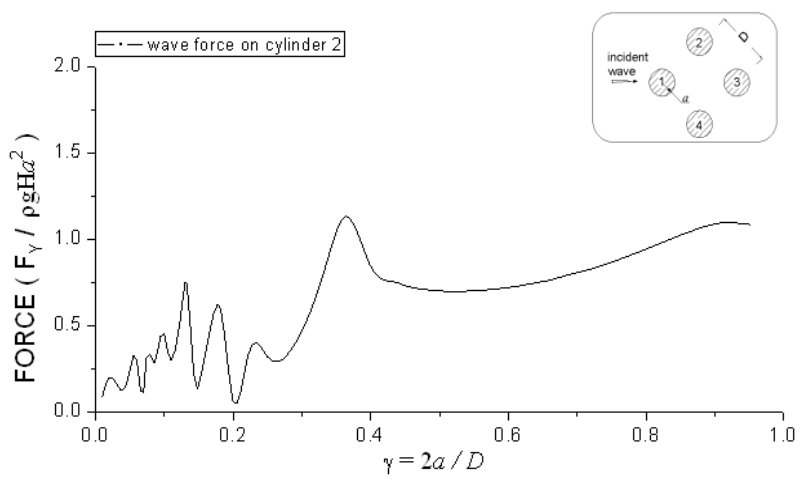

Fig. 8 Wave forces in $y$-direction on cylinder 2 in lozenge array for $k a=1.0$
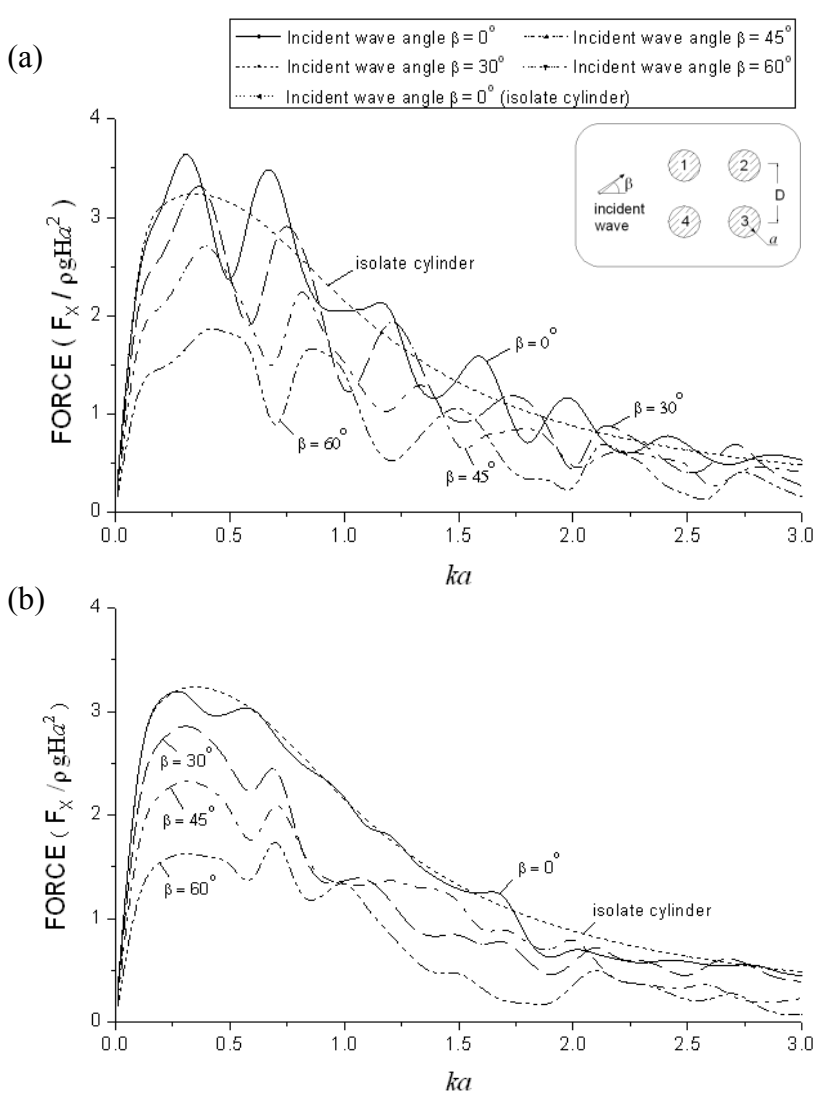

(c)

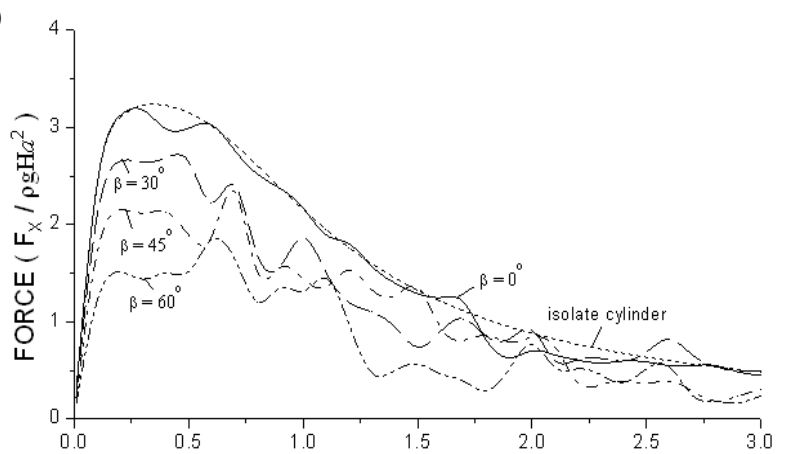

(d)

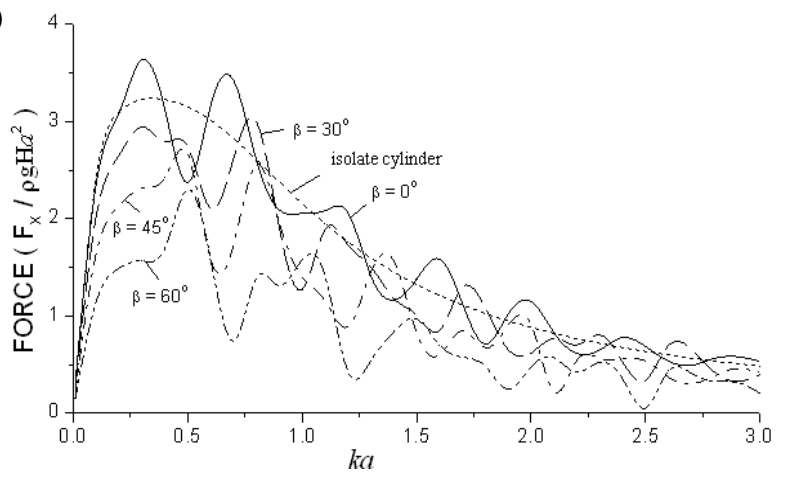

Fig. 9 Wave forces in $x$-direction on each cylinder in square array versus wave number $k a$ and incident wave angle $\beta$ for $D / a=8, h / a=10$ : (a) Wave force on cylinder 1; (b) Wave force on cylinder 2; (c) Wave force on cylinder 3; (d) Wave force on cylinder 4 
The Boundary Element Analysis of Wave Force acting on Multiple Cylinders

(a)

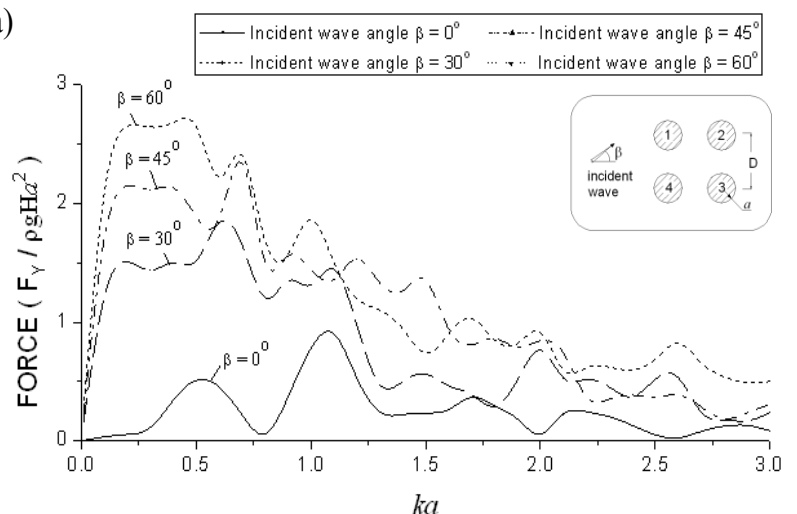

(b)

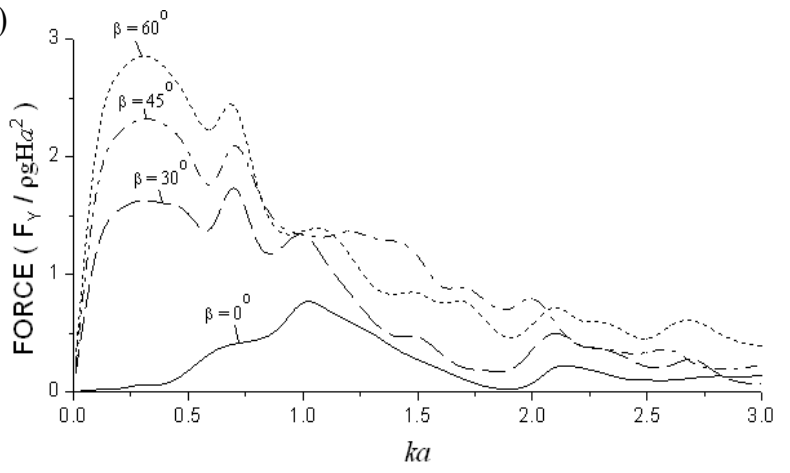

(c)

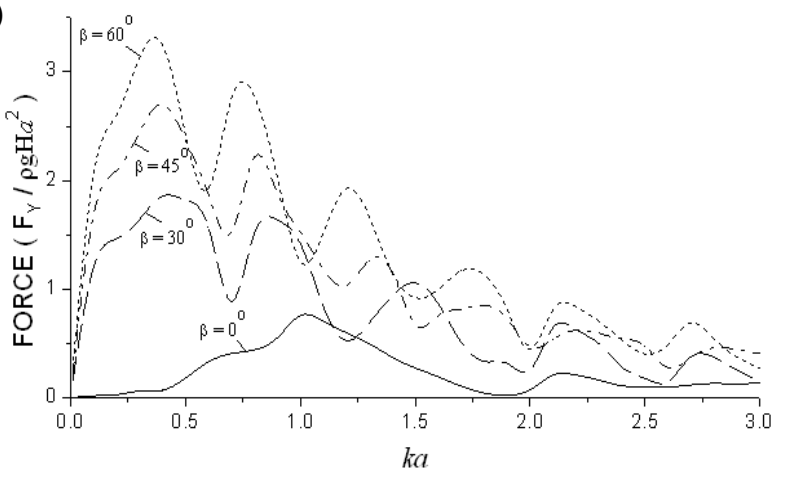

(d)

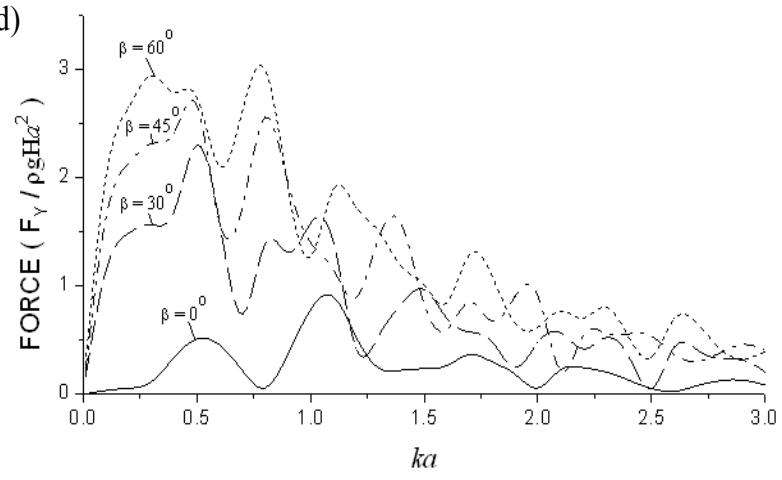

Fig. 10 Wave forces in $y$-direction on each cylinder in square array versus wave number $k a$ and incident wave angle $\beta$ for $D / a=8, h / a=10$ : (a) Wave force on cylinder 1; (b) Wave force on cylinder 2; (c) Wave force on cylinder 3; (d) Wave force on cylinder 4

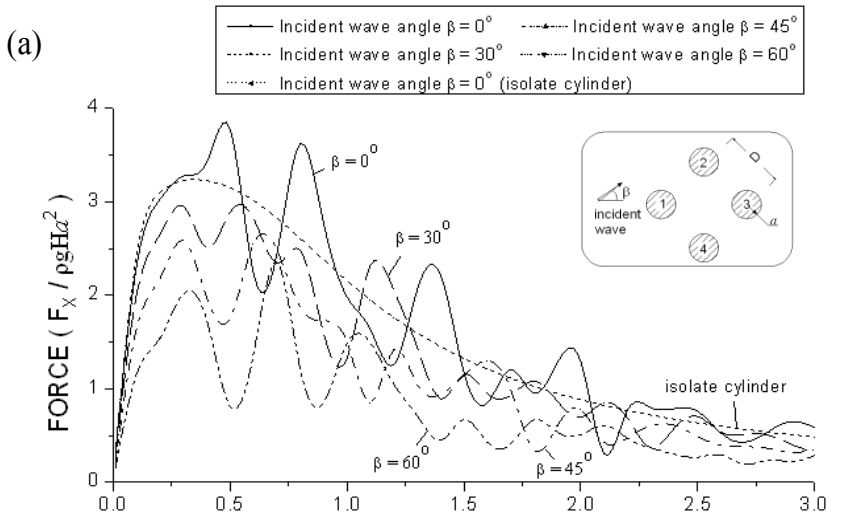

(b)

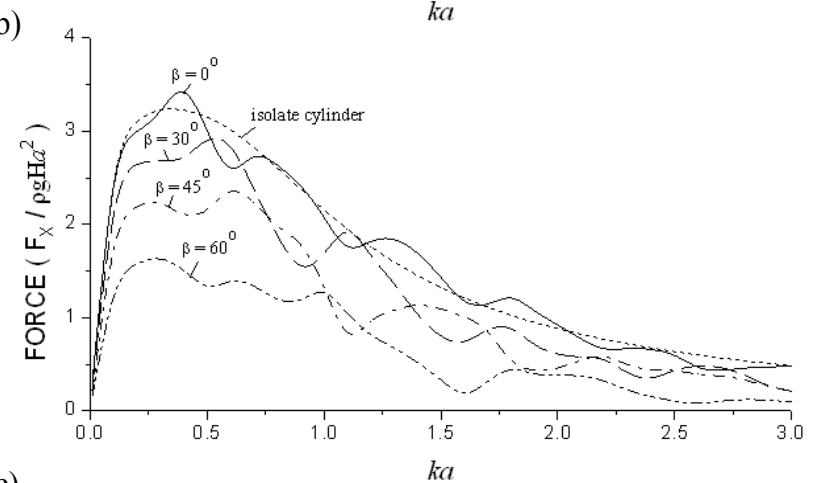

(c)

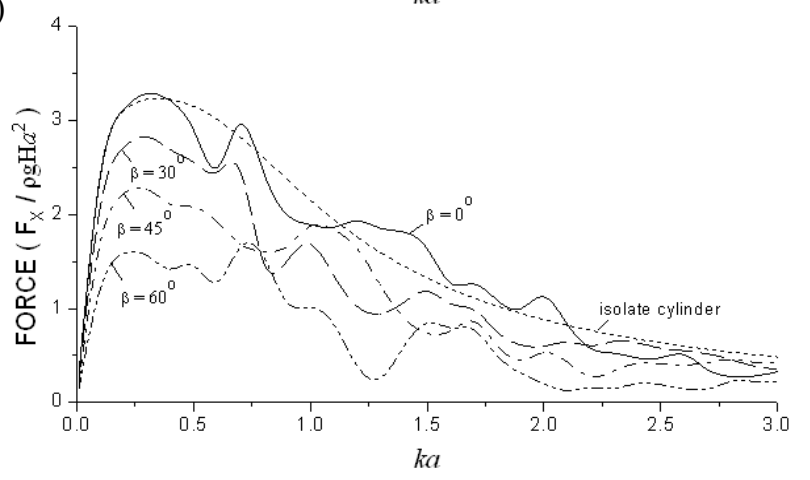

(d)

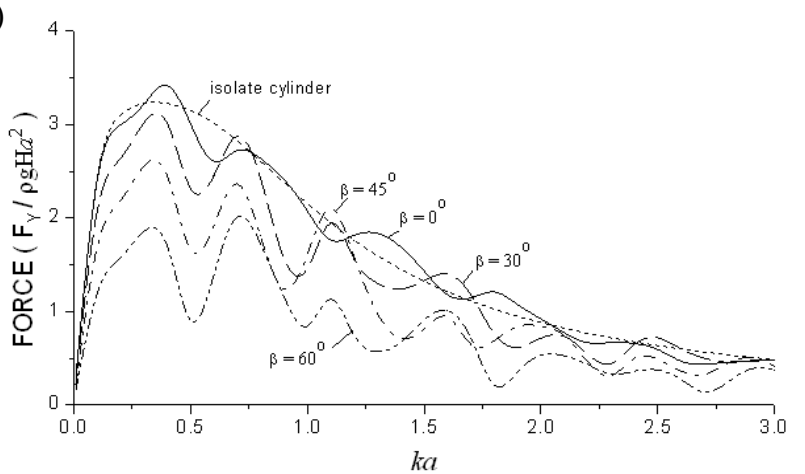

Fig. 11 Wave forces in $x$-direction on each cylinder in lozenge array versus wave number $k a$ and incident wave angle $\beta$ : (a) Wave force on cylinder 1; (b) Wave force on cylinder 2; (c) Wave force on cylinder 3; (d) Wave force on cylinder 4 

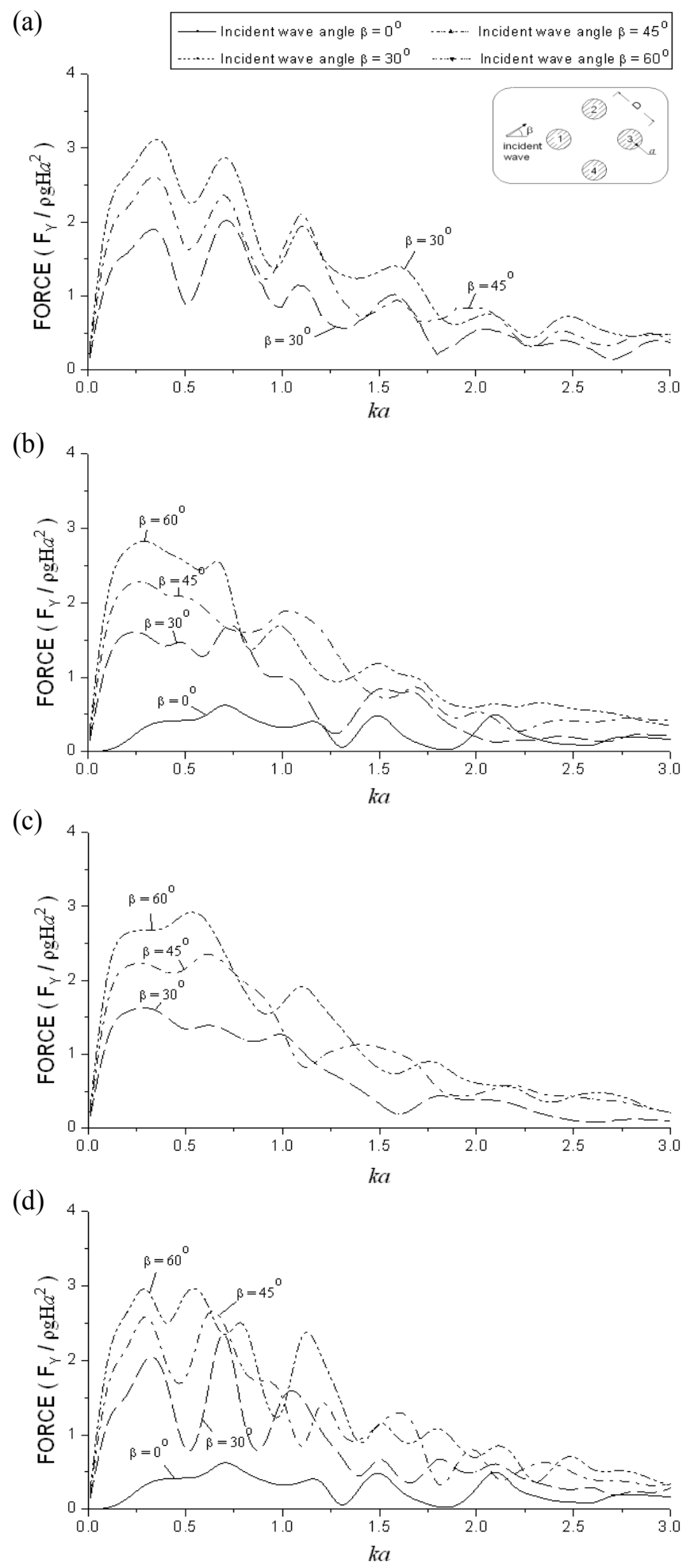

Fig. 12 Wave forces in $y$-direction on each cylinder in lozenge array versus wave number $k a$ and incident wave angle $\beta$ : (a) Wave force on cylinder 1 ; (b) Wave force on cylinder 2; (c) Wave force on cylinder 3; (d) Wave force on cylinder 4
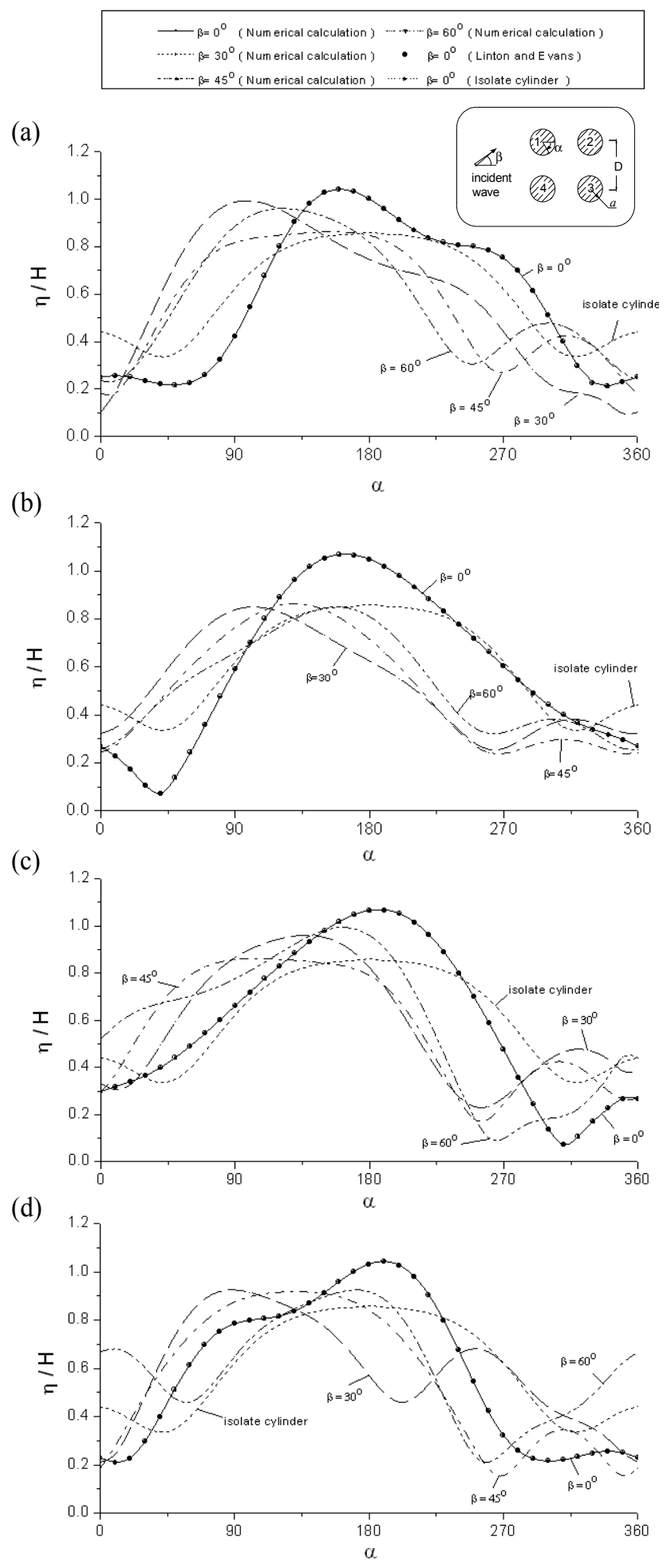

Fig. 13 Run-up on each cylinder in square array for $D / a=8, \quad h / a=10, \quad k a=1.0$ at various incident wave angles: (a) Run-up on cylinder 1; (b) Run-up on cylinder 2; (c) Run-up on cylinder 3; (d) Run-up on cylinder 4 


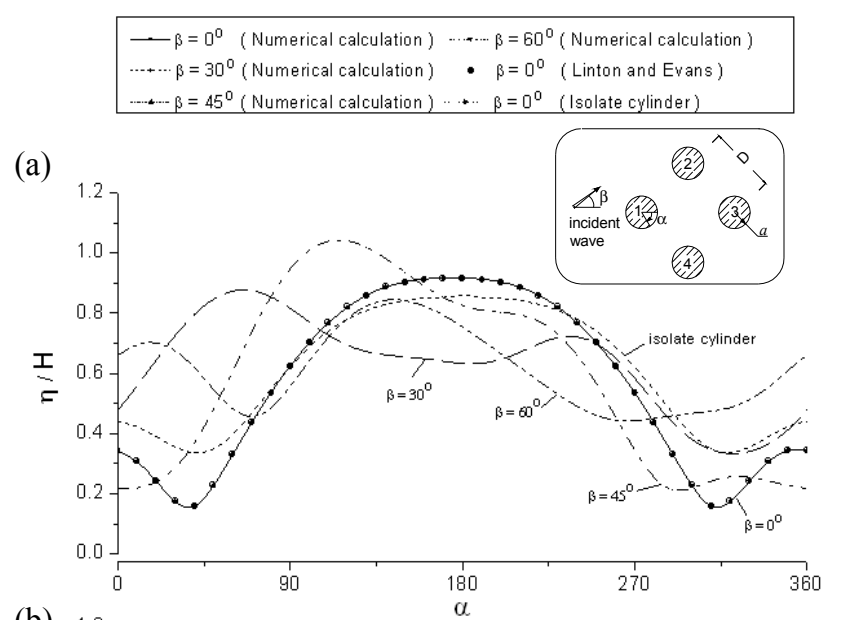

(b)

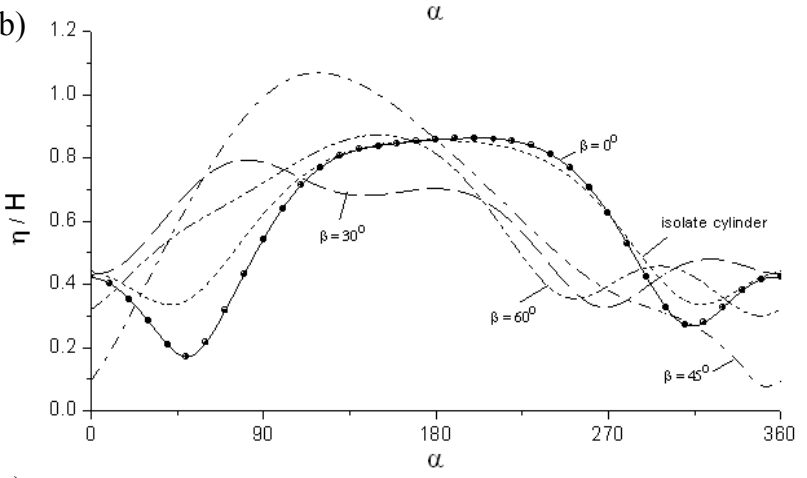

(c)

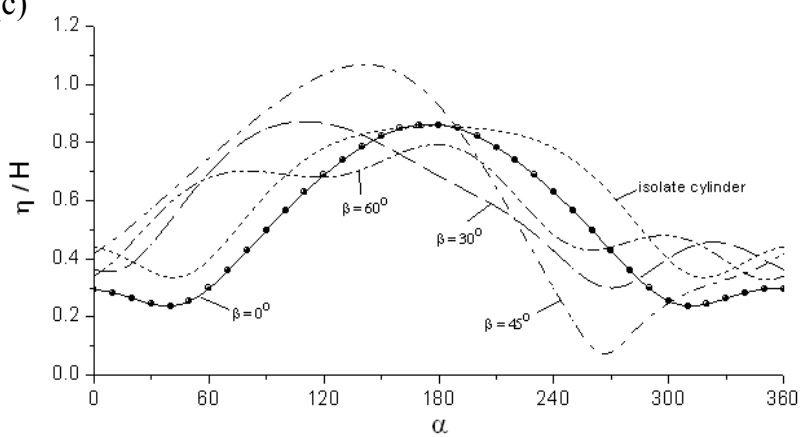

(d)

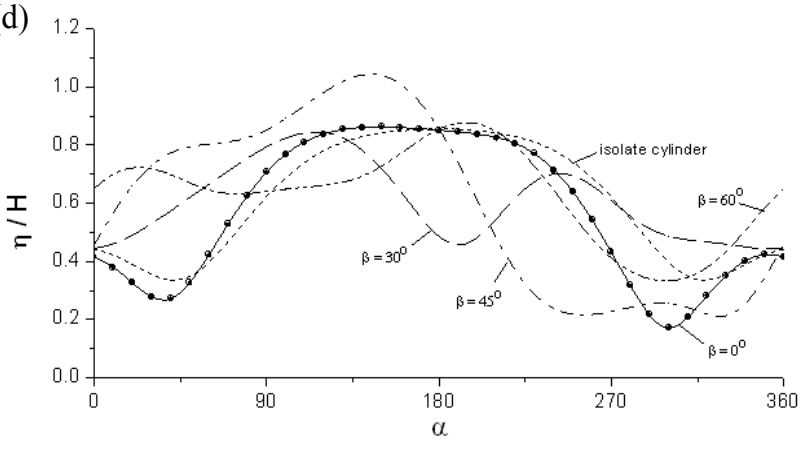

Fig. 14 Run-up on each cylinder in lozenge array for $D / a=8, \quad h / a=10, \quad k a=1.0$ at various incident wave angles: (a) Run-up on cylinder 1; (b) Run-up on cylinder 2; (c) Run-up on cylinder 3; (d) Run-up on cylinder 4

isolate cylinder. The amplitude of oscillation is large as the ratio $\gamma>0.2$. As the distance approaches infinity, the wave force on each cylinder tends to that of an isolate cylinder.
The numerical results of this study show strong agreement with those of Chakrabarti(1978).

Fig. 9 to Fig. 12 present the wave forces in $x-$ and $y$ -direction acting on four cylinders in square and lozenge array versus the wave number $k a$ for $D / a=8, h / a=10$ at different incident wave angles $\beta=0^{\circ}, 30^{\circ}, 45^{\circ}$, and $60^{\circ}$. The figures show that the wave forces on the front cylinders extremely larger than the wave forces on the isolate cylinder and the rear cylinders because of the interaction among the cylinders and the shielding effect. Also the figures show that the wave forces in $x$-direction tend to decrease and the wave forces in $y$-direction tend to increase graduallyas the incident wave angle increases.

Fig. 13 and Fig. 14 present the run-up on each of four cylinders in square and lozenge array for $D / a=8$, $h / a=10, k a=1.0$ at different incident wave angles $\beta=0^{\circ}$, $30^{\circ}, 45^{\circ}$, and $60^{\circ}$. The run-up on the isolate cylinder is also plotted for comparison. The results show that due to the interaction between the cylinders, the run-up profile on each cylinders is quite different from that of the isolate cylinder, and in most cases the maximum run-up on the front cylinder is higher than that of the isolate cylinder because of the interaction of the cylinders. The maximum run-up on the front cylinder are higher than that on the rear cylinders because of the shielding effect. Also, the results of this numerical analysis method show a strong agreement with those of Linton and Evans (1990).

\section{Conclusion}

The wave pressures and wave forces acting on each of four vertical circular cylinders in square and lozenge array are derived by using boundary element method.

The results obtained from this numerical analysis method for a row of seven cylinders normal to the incident waves show strong agreement with the experiment data obtained by Sobierajski(1970) and the results of Chakrabarti (1978).

The wave forces acting on four cylinders in square and lozenge array are calculated and presented. Also, the effects of various parameters such as the cylinder spacing, the incident wave angle and the wave number on the hydrodynamic loads are considered and presented in this paper. The computed results show that as the distance between the cylinders increases, the wave force on each cylinder in the cylinder group oscillates around the wave force on an isolate cylinder. The amplitude of oscillation is large as the ratio $\gamma>0.2$. 
In addition, the run-up on each of four cylinders in square and lozenge array are calculated. The numerical results of this study show that the run-up profile on each cylinders is quite different from that on the isolate cylinder due to the interaction between the cylinders. The computed results also show strong agreement with the results of Linton and Evans(1990).

This numerical analysis developed by boundary element method could be used broadly for the design of various offshore structures to be constructed in coastal zones in the future.

\section{References}

[1] Chakrabarti, S. K.(1978), "Wave forces on multiple vertical cylinders." Journal of Waterway, Port, Coastal and Ocean Division, Vol. 104, No. 2, pp. 147-161.

[2] Chakrabarti, S. K.(2000), "Hydrodynamic interaction forces on multiple-moduled structures." Ocean Engineering, Vol. 27, No. 10, pp. 1037-1063.

[3] Kagemoto, H. and Yue, D. K. P.(1986), "Interactions among multiple three-dimensional bodies in water waves: an exact algebraic method." Journal of Fluid Mechanics, Vol. 166, pp. 189-209.

[4] Kim, M. H.(1992), "Interaction of waves with N vertical circular cylinders." Journal of Waterway, Port, Coastal, and Ocean Engineering, ASCE, Vol. 119, No. 6 pp. 671-689

[5] Kim, N. H., Park, M. S. and Yang, S. B.(2007). "Wave force analysis of the vertical circular cylinder by boundary element method." KSCE Journal of Civil Engineering, Vol. 11, No. 1, pp. 31-35.

[6] Kim, N. H. and Cao, T. N. T.(2008a), "Wave force analysis of the two vertical cylinders by boundary element method." KSCE Journal of Civil Engineering, Vol. 12, No. 6, pp. 359-366.

[7] Kim, N. H. and Cao, T. N. T.(2008b), "Wave force analysis of the three vertical cylinders in water waves." Journal of Navigation and Port Research International Edition, Vol. 32, No. 7, pp. 543-552.

[8] Linton, C. M. and Evans, D. V.(1990), "The interaction of waves with arrays of vertical circular cylinders." Journal of Fluid Mechanics, Vol. 215, pp. 549-569.

[9] MacCamy, R. C. and Fuchs, R. A.(1954), "Wave forces on piles: A diffraction theory." Tech. Memo, No. 69, US Army Coastal Engineering Research Center.

[10] Ohkusu, M.(1974), "Hydrodynamic forces on multiple cylinders in waves." Proceedings of International
Symposium on Dynamics of Marine Vehicles and Structures in Waves, London, Paper 12, pp. 107-112.

[9] Sobierajski, E.(1970), "Hydrodynamic interpretation of the gravity wave action on piles and columns." RozprawyHydrotechiczne, Vol. 26, pp. 37-64(inPolish).

[10] Spring, B. H. and Monkmeyer, P. L.(1974), "Interaction of plane waves with vertical cylinders." Proceedings of 14th international conference on coastal engineering, Copenhagen, Denamrk, Vol. 107, pp. 1828-1847.

[11] Twersky, V.(1950), "Multiple scattering of radiation by an arbitrary configuration of parallel cylinders." Journal of the Acoustical Society of America, Vol. 24, pp. 42-46.

[12] Williams, A. N. and Li, W.(2000), "Water wave interaction with an array of bottom-mounted surface-piercing porous cylinders." Ocean Engineering, Vol. 27, No. 8, pp. 841-866.

\footnotetext{
Received 8 August 2012

Revised 23 September 2012

Accepted 24 September 2012
} 